\title{
EMPÍRICO Y CIENTÍFICO: DOS FACES DE LA EDUCACIÓN MÉDICA
}

\author{
Empirical and scientific: two faces of medical education
}

\author{
Luis Fernando Molas Lopez ${ }^{1}$; Rochana Basso²; Fernando Gabriel Molas ${ }^{3}$
}

\begin{abstract}
${ }^{1}$ Médico cirujano obstetra del Sistema de Salud del Paraguay - Universidad Católica Nuestra Señora de la Asunción - Paraguay. E-mail: fernando.molas@gmail.com

${ }^{2}$ Bel en Derecho. Experto en Derecho Civil. Experto en Derecho Notarial. Mestranda en Educación URI/Frederico Westphalen. E-mail: bassorochana@gmail.com

${ }^{3}$ Académico del Carrera de Medicina de la UMAX-Paraguay.E-mail: estudos2210@gmail.com
\end{abstract}

Data do recebimento: 04/07/2020 - Data do aceite: 14/10/2020

RESUMEN: Este artículo versa sobre las interfaces del sentido común y la ciencia médica. Busca señalar algunas colaboraciones empíricas en el tratamiento de la salud, especialmente con respecto a la dicotomía cultural existente entre el estándar de tratamiento médico y el empirismo presente en las prácticas cotidianas. Las inferencias realizadas están vinculadas a la producción y socialización del conocimiento relacionado con las prácticas de salud por las cuales pasan las verdades de la curación y a través de curación.

Palabras-clave: Conocimiento. Sentido común. Ciencia médica.

\begin{abstract}
This article deals with the interfaces of common sense and medical science. It seeks to point out some empirical collaborations in health treatment, especially with regard to the cultural dichotomy existing between the standard of medical treatment and the empiricism present in everyday practices. The inferences made are linked to the production and socialization of knowledge related to health practices through the truths of healing and through healing.
\end{abstract}

Keywords: Education. Empiricism. Medical science. Methodology. 


\section{Introducción}

En este artículo se busca demostrar alineaciones entre los diferentes conocimientos del área de la salud y como esos pueden ser regidos por la educación en virtud de la salud para la sociedad. Empezamos desde el principio de que el saber empírico es aquel adquirido en base a las experiencias y vivencias cotidianas, teniendo como base las percepciones de los sentidos humanos. Sin embargo, el saber científico proviene de la investigación y respectivo análisis minucioso de eventos que, al ser comprobado y reconocido, puede ser transformado en teorías.

Así, ante la precarización del sistema de salud, en nivel mundial, que conduce a las personas a buscar formas alternativas para poder recuperar su salud, se busca la reflexión sobre las posibilidades informales de alcanzar la cura, aclarando el discernimiento sobre si el saber empírico asiste o interfiere al saber científico de la práctica médica tradicional y presentar a la educación como vector que conecta las extremidades de las diferentes sapiencias.

Desde tiempos remotos, en la cultura precolombina los conocimientos en áreas de salud estaban bien arraigados y definidos en el ámbito medicinal autóctono, desde los aztecas al norte, pasando por los mayas, incas, llegando a los guaraníes, sociedad indígena que se destacó en el arte de las hierbas medicinales como ningún otro, incluso siendo esos conocimientos llevados al viejo mundo como parte de sus valiosos hallazgos. La ciencia médica farmacológica en la actualidad sigue haciendo uso de sus actualmente demostrados efectos conocidos, procesados en innumerables estudios científicos e incluso comercializados ya con todo el aparataje resultante de las demostraciones basadas en estudios metodológicos, dando así una oferta a la sociedad sin que esta desconozca esa misma medicación en su forma botánica.

\section{Conceptos}

El empirismo surge como una reacción a la razón, de tal forma que los empiristas defienden la experiencia sensorial, los conocimientos de las ideas y de las percepciones que los sentidos aprenden del mundo exterior, para consolidar que el conocimiento adquirido durante la vida es resultado de experiencias sensoriales (TARTUCE, 2006). Así, el saber empírico puede ser definido a partir de la doctrina o sistema que sólo reconoce la experiencia como: "[...] conjunto de conocimientos adquiridos solo por la práctica" (FERREIRA, 2011, p. 259).

Mientras que el saber científico proviene de la confirmación por medio de estudios testificados por metodologías que comprueban la aptitud de sus acciones. Los decires de Gerardth y Silveira (2009) es entendido como objetivo, metódico, pasible de demostración y comprobación. Ese saber está fundamentado en análisis de hechos comprobados, basados en la observación y experimentación para comprobación de determinados temas. De esa manera, se presenta en carácter provisional, una vez que puede ser contestado, enriquecido y reformulado (FONSECA, 2011, p. 11).

\section{El sentido común y sus matices en el tratamiento}

Una de las primeras manifestaciones empíricas, vinculadas a la salud, desciende de la religión que, según Santos et al. (2004), expresa una búsqueda de vínculos entre personas y el divino, causados por el sistema de salud precario que llevó a una gran cantidad de la población en búsqueda de formas alternativas para restablecimiento de su bienestar. 
Existe un contexto de vivencias y experiencias que forman una estructura suficiente para arrojar al hombre en la búsqueda de alivio a sus dolores, desde una simple espina en el pie, que le amormaba, hasta una situación más compleja de morbilidad.

En el Medioevo, era dada al sacerdote la función de curar los males que cometían al hombre, pues se tomaba como verdad que las enfermedades eran consecuencia de ofensas direccionadas a los seres divinos. También se creía que sólo el sacerdote logra prestigio suficiente ante los seres divinos y puede interceder junto a los seres a favor de los hombres, bendiciendo, para así tener una "pseudo-cura". Juntamente con las bendiciones y oraciones para la curación del enfermo, el sacerdote gozaba de varios otros métodos para expurgación de la punición, como sangría, laxantes, vómitos, alucinógenos y hasta mutilaciones de parte del cuerpo.

Un poco más adelante, en el siglo XIX, por influencia de la religión, se creía en la relación entre las enfermedades anatómicas y psicológicas, restableciendo la creencia de que el sufrimiento sería un castigo por la falta de moral, del cual el individuo recibiría su punición (VAZ et al., 2007). En aquel hito el objetivo de las iglesias era de acoger y regar la semilla de la ilusión emascarada por la protección, dando así un falso sentido a sus vidas y de esa manera mantener su rebaño ignorante bajo su control.

La religión nasce, entonces, de la necesidad de los seres humanos en interpretar y hallar algunas explicaciones para que los fenómenos de la naturaleza, incluso de su propia existencia. La falta de conocimientos científicos, que ayuda al hombre a entender o comprender a sí mismo y al mundo en que vivía, lo llevaba a creer en una fuerza superior, fuera del ser humano, que ordenaba y hasta la ocurrencia de las cosas en este mundo, para las cuales él no tenía explicación o no conseguía comprender. Así, hallaba explicaciones para los fenómenos de su entorno, la existencia de seres misteriosos, mágicos, capaces de controlar el clima, los volcanes, temporadas de caza, entre otros. Al mismo tiempo en que buscaba entender el mundo en el entorno a sí mismo. El hombre tenía aún más otras preocupaciones: la preservación de su propia vida; de ahí transcurre la necesidad de buscar la curación para enfermedades que asolaban su existencia.

Hoy por hoy, la mayoría de las iglesias actúa en el campo de la salud y ofrece ayuda emocional y cura, interfiriendo en la manera en que las personas afrontan, aceptan las enfermedades y buscan el restablecimiento de la salud. Eso hace que el fiel se vuelva un "cliente" de la iglesia, o sea, "un consumidor" en la búsqueda de servicios ofrecidos por esas instituciones. Las curas pasan a tener un carácter de medicina popular y los milagros pasan a ser un consenso divulgado por los medios. (MELLO; OLIVEIRA, 2013). En la actualidad, las prácticas de curas, por medio de los cultos religiosos, están siendo muy reconocidos por acción como agencias terapéuticas entre las comunidades (SANTOS et al., 2004). De esa manera, de acuerdo con Mello y Oliveira (2013), una de las mayores características culturales en la sociedad brasilera es una creencia en espíritus donde la mayoría de las personas cree en una acción de lo sagrado en la prevención y en la curación de las enfermedades que impactaban, en su perpetuidad, debilitándolos.

En el campo de la fitoterapia, un ejemplo del uso del saber empírico y dado por Santos et al. (2012), cuando relata sobre el uso popular de plantas como pata de vaca (Bahuinia $\mathrm{sp}$ ), aceituna lila (Syzguim jambalanum DC.) y la insulina vegetal (Cissus sicyoides L.) para el tratamiento de la diabetes. Carvalho et al. (2005) constatan con Santos e incluyen el ajo, el aloe vera, la carqueja, y la cebolla, el eucalipto, la grabiola, el melón San Cayetano 
y la granada como plantas que también tienen perfil antidiabético.

El saber empírico puede ser considerado una de las formas de medicinas alternativas. Se trata de modelos de muy diversos origenes y de diferentes rasgos culturales: filones heterodoxos de la misma medicina occidental, como la homeopatía o la pranoterapia; survivals de viejas tradiciones herbolarias cultas o populares; como también de producciones semiindustriales de instrumentos de 'defensa mágica' comercializadas por intermedio de redes publicitarias y canales de distribución a domicilio; formas actualizadas de sanadores; técnicas de prevención o terapia, tales como: el yoga, la acupuntura, la moxibustión o el shia-tzu -procedentes de las grandes culturas médicas del Extremo Oriente. (PERDIGUERO; COMELLES, 2000, p. 40-42).

De esa manera, se podría decidir que el sentido común y sus matices en el tratamiento abrirán espacios para que la ciencia halle la curación para las patologías tanto físicas como psíquicas, y también lo hizo posibles nuevas formas de medicina, conocidas como alternativas.

\section{La ciencia médica y el empirismo}

A partir del siglo $\mathrm{XX}$ se puede percibir una evolución en el concepto de salud. Se quiere cargar lo inicialmente como ciencia de la enfermedad. Tal noción estaría en una íntima relación con el modelo biomédico (en la cual la relación médico/paciente está tan próxima de una visión bancaria de educación - que se entiende como aquella en que el maestro enseña y el alumno solamente repite sin permiso para reflexión), en una visión de hombre/máquina en que la propia enfermedad era enfocada en una causalidad linear, únicamente, biológica (MACHADO, 2006). La salud deja de ser solamente la ausencia de enfermedad, para ser entendida como un estado de completo bienestar físico, mental, social y espiritual, en constante cambio a lo largo de la vida.

Aunque el relatorio Lalonde (1974), indicaba la importancia del ambiente exterior de las decisiones individuales (comportamientos o estilos de vida) en la salud (SAKELLARIDES, 2005; TURA, 2009), el cambio paradigmático en el concepto de salud se evidencia más específicamente en 1978 cuando la OMS (Organización Mundial de la Salud) realizó el Alma-Ata una conferencia internacional sobre cuidados básicos de salud.

Reafirma, como derecho fundamental, la salud pasó a ser considerada como un estado de "completo bienestar físico, mental y social y no sólo ausencia de enfermedades o dolencias" (OMS, 1978, p.1). Por lo tanto, ese concepto no niega la existencia de enfermedad; sólo define un estado positivo, que se descentró de la misma; es dinámico, ya que va sucediendo alteraciones a lo largo de la vida de los individuos, y que, por eso, no se tienen como condición definida en la medida en que se va conquistando individualmente (SERRÃO, 2010).

En la conferencia superior, fue reprobada la asimetría en salud que se vive dentro de los países centrales, entre éstos y los periféricos, y que se refleja en términos económicos, responsabilizando a los gobiernos por la salud de sus poblaciones. La evolución del concepto de salud también fue probada en varios documentos producidos en la conferencia de Ottawa (OMS, 1986) perdurando hasta Nairobi (WHO, 2009). En la primera de las conferencias surgió como la creciente expectativa en salud a hacer mejoras significativas que se registraron en salud de poblaciones, sobre todo de los países centrales que implementan las recomendaciones defendidas en Alma-Ata.

La salud tera como un recurso para a vida y no como un fin en la vida; se centra, sí, en la persona en la comunidad. 
Más recientemente, surge como concepto de salud global, refiriéndose "a los impactos trasnacionales de la globalización sobre los determinantes y los problemas de salud que están para más alla del control de cada nación" (SMITH; TANG; NUTBEAM, 2006, p. 342). En la llamada para la acción en Nairobi, con el título Promocionar una salud y un desarrollo: romper los espacios de implementación (WHO, 2009). Se reiteró la importancia de la capacitación individual y comunitaria, al fortalecimiento del trabajo intersectorial y la necesidad de inscribir la promoción de salud en agendas políticas, en su papel en el avance integrado de las naciones.

En ese lapso, es necesario retomar las evidencias empíricas que llevan a la población a adoptar prácticas de curas por cuenta, y a veces, la ineficiencia de las políticas públicas, en el área de salud, que no hayan conseguido alcanzar aquellos que más necesitan de acciones en salud, dando curso así a diversos mecanismos para la recuperación de la misma, como es el caso de uso de fitoterápicos.

Pese a que existan varias investigaciones sobre las plantas medicinales, la escasez de estudios clínicos más profundos todavía persiste. Es importante entender que algunas especies vegetales pueden producir efectos tóxicos, resultando en el efecto deseado hipoglicemia; sin embargo, no como un fenómeno terapéutico pero sí tóxico (SANTOS et al., 2012).

Es extremadamente importante resaltar que, en la medicina herbaria, pueden actuar como forma opcional de terapéutica, llevando en consideración un menor coste. Por lo tanto, la mayoría de los casos de problemas de salud, no se puede excluir los fármacos que todavía predominan en el control de enfermedades de la civilización post moderna. Por eso, es interesante subrayar que los beneficios de terapia, con uso de especies vegetales, se agregan a la terapia convencional (SANTOS et al., 2012).
Por tanto existen pruebas científicas sobre la efectividad en el tratamiento utilizando plantas medicinales de uso popular, la carencia de estudios clínicos más profundos todavía persiste, visto que algunas especies vegetales pueden tener efectos imprecisos dentro de patologías específicas, interfiriendo en el resultado terapéutico científico, se halla en forma positiva, o en forma negativa (SANTOS et al., 2012).

La Organización Mundial de la Salud (OMS) reconoce la importancia de la fitoterapia y propone ser una opción accesible a poblaciones de países en desarrollo, como Brasil, debido a su coste reducido (REZENDE; COCCO, 2002).

El modelo de atención de salud, a veces, no está siendo eficaz contra la complejidad de los problemas de salud de la población, porque no considera que hay características individuales en cada región. Las plantas medicinales, o medicina de sentido común, son utilizadas desde miles de años y sus conocimientos curativos son pasados de generación en generación, sobrepasando barreras continentales, siguiendo reconocidos por la Organización Mundial de la Salud; con salvedades para el uso racional de esa práctica (BOCHNER et al., 2012). Ese saber popular es una práctica frecuente de la población; por lo tanto, necesita de más estudios sobre qué patologías podrían ser acusadas, no sustituyendo la medicina científica en diversas enfermedades, como las metabólicas e infecciosas, por ejemplo.

La medicina científica se vale de mediciones derivadas del conocimiento cultural y empírico de la población, cuyas plantas medicinales han sido estudiadas cuidadosamente y, a partir de ahí, extraído el principio activo para curar (BALDAUF et al., 2009). Por ser de fácil cultivo en casa, y por presentar un bajo coste en comparación a medicaciones industrializadas, el advenimiento de esa nueva forma de consumir, lo más natural 
posible, la medicina popular volvió a ser un punto importante de discusiones.

\section{La educación como el dialogo de los saberes}

Como la salud evolucionó conceptualmente a partir del siglo XX, la educación también rebuscó, en el último siglo, profundos cambios, tanto en el plan conceptual como en las prácticas decurrentes, teniendo como origen las trasformaciones por las cuales la humanidad experimentó en niveles económico, político y social.

La educación deja de ser preceptora de niños y jóvenes, solamente, pautada por asimilación de conocimientos, y asume una postura más integradora, centrada en la creación de condiciones que permiten a los individuos se desarrollar holísticamente en sus dimensiones, en permanente integración con otros, caracterizada por Freire (1976) con educación libertadora, que pasa por una perspectiva más integradora, creadora de condiciones que permitían a las personas evolucionar en sus dimensiones.

En la mitad del siglo XIX, la ONU (Organización Mundial de la Salud) creó la Declaración Universal de los Derechos del Hombre, que concibe la dignidad humana como un valor inherente a la propia humanidad, fundamento de todos los derechos y libertad fundamentales, alcanzables por la educación, y se afirma el derecho a la educación con vista a la expansión de personalidad individual (ONU, 1948). Varios cambios $\mathrm{y}$ adecuaciones emergieron en secuencia; entre ellos están las diversas conferencias internacionales de educación de mayores, promocionada por UNESCO.

Bajo ese contexto, con atención al crecimiento económico y el bienestar social, el mundo empieza a aceptar y asumir la insepa- rable relación entre educación y desarrollo, priorizando la educación enfocada a los conocimientos profesionales a los cambios tecnológicos.

Ocurre, así, una ruptura con el modelo educacional, y la educación se vuelve al desarrollo integral del hombre, potenciando su capacidad y su participación en el desarrollo social. El hecho de ver que cada individuo es fructo de su propia historia de vida se empieza a notar la educación como un proceso que objectiva a crear condiciones de desarrollo de todas las capacidades de los individuos.

En el siglo XXI la educación agrega a su concepto la noción de responsabilidad individual y comunitaria a fin de capacitar a los individuos para relacionarse con transformaciones sociales. Se hace necesario, por lo tanto, que la sociedad fuera incluida en el proceso no destituido de la responsabilidad gubernativa, estabilizando la educación en respeto por los derechos humanos como forma de alcanzar un adelanto equitativo $y$ (ecológicamente) sustentable.

También se conectó el papel de la educación con la equidad de acceso a la salud, enfocándose la multidimensional que exige atención constante y de continuo desenvolvimiento" (UNESCO, 2010 p.12), conduciendo, así, procesos educativos que llevan a aprendizajes.

Es oportuno salientar que los varios documentos resultantes de las conferencias en educación están responsabilizando a los gobernantes en creaciones de políticas educativas cómplices de las nuevas concepciones preconizadas.

\section{Consideraciones Finales}

Hipócrates buscó transmitir las técnicas de medicina, y Aristóteles trajo la base primordial del conocimiento que son las 
sensaciones y los contactos inmediatos con el mundo. Impregnada en la memoria, la repetida marca de esa sensación constituye experiencias que, a partir de percepciones individuales, avanzan para asociaciones generales. La ciencia, entretanto, busca el desconocimiento y comprueba con la demostración. De otro lado, el sentido común representa los saberes que todavía no fueron absorbidos por el conocimiento científico sistematizado, representado por las experiencias prácticas y sin la necesidad previa de bases teóricas.

Comúnmente se presenta la génesis de la ciencia en términos de una evolución linear, que, partiendo de los mitos, pasa por la filosofía, hasta alcanzar el Status de la ciencia experimental. En ese acceso, todavía, no se revela el contexto en que tales transiciones son construidas.

Frente a eso, se puede afirmar que, por medio de la educación, es posible notar la estrecha relación entre ciencia médica y el sentido común. Muchos medicamentos fueron desarrollados a partir de estudios profundos sobre el conocimiento empírico, demostrando, así, la importancia del conocimiento del sentido común en la práctica de la ciencia médica.

Así, salud y educación pasan a ser planteadas con dos faces de un mismo proceso que hacen que las prácticas educativas vueltas a la salud exibieran mejorías para la sociedad en su total, pues es por medio del dialogo entre los saberes que se vuelven factibles las técnicas, asociando las prácticas médicas con el sentido común.

\section{REFERENCIAS}

BALDAUF, C.; KUBO, R. R; SILVA, F.; IRGANG, B. E. "Hirvió, quemó el ser de la hierba": conocimiento de expertos locales en plantas medicinales en la región sur de Brasil. Revista Brasileña de Plantas Medicinales, v.11, n.3, p.282-291, 2009.

BOCHNER, R.; FISZON, J. T.; ASSIS, M. A.; AVELAR, K. E. S. Problemas asociados con el uso de plantas medicinales vendidas en Mercadão de Madureira, municipio de Río de Janeiro, Brasil.

Revista Brasileña de Plantas Medicinales, v. 14, n. 3, p. 537-547, 2012.

FERREIRA, A. B. H. Mini Aurélio Siglo: el mini-diccionario de la lengua portuguesa. 4. ed. Río de Janeiro: Nueva Frontera, 2011.

FONSECA, J. J. S. Ala naturaleza de la ciencia y la investigación científica. En: FONSECA. Metodología de la investigación científica. Fortaleza: UEC, 2011.

FREIRE, P. Pedagogía de los oprimidos. Rio de Enero: Paz y Tierra, 1976.

GERARDTH, T.; SILVEIRA, D. Métodos de investigación. Puerto Alegre: Compañia de publicidad de la UFRGS, 2009.

LALONDE, M. A New Perspective on the Health of Canadians. Ottawa: Canadian Ministry of National Health and Welfare. 1974.

MACHADO, J. Perspectiva antropológica do ensino da medicina. Acção Médica, Lisboa, v. LXX, n. 1, p. 16-22, 2006.

MELLO, M. L.; OLIVEIRA, S. S. Salud, religión y cultura: un diálogo basado en las prácticas afrobrasileñas. São Paulo. Salud y sociedad, v. 22, n. 4, p. 1024-1035, 2013. 
OMS - ORGANIZACIÓN MUNDIAL DE LA SALUD. Declaración de Alma-Ata: Primera Conferencia Internacional sobre atención primaria de salud. Ginebra, 1978.

OMS - ORGANIZACIÓN MUNDIAL DE LA SALUD. Carta de Ottawa para la promoción de la salud: Primera Conferencia Internacional sobre promoción de la salud. Ginebra. Genebra, 1986.

ONU - ORGANIZACIÓN DE LAS NACIONES UNIDAS. Declaración Universal de los Derechos Humanos. Nueva York, 1948. Disponible en: http: //www.gddc. pt / human-rights/internationaltexts-dh / tidhuniversais / cidh-dudh.html. Acceso en: 12 abr. 2020.

PERDIGUERO, E.; COMELLES, J. M. Medicina y cultura. Estudios entre la antropología y la medicina. Ediciones Bellaterra. Barcelona. 2000.

REZENDE, H. A; COCCO, M. I. M. El uso de fitoterapia entró en la vida cotidiana de una población rural. Revista de la Escuela de Enfermería de la USP, v. 36, n. 3, p. 282-288, 2002.

SAKELLARIDES, C. De alma a Harry: crónica da democratização da saúde. Coimbra: Almedina, 2005.

SANTOS, E. C.; KOLLER, S. H.; PEREIRA, M. T. L. N. Religión, salud y curación: un estudio entre neopentecostales, Brasília. Psicologia, Ciência e Profesion, v. 24, n. 3, p. 82-91, 2004.

SANTOS, M. M.; NUNES, M. G. S.; MARTINS, R. D. Uso empírico de plantas medicinales para tratar la diabetes, Botucatu. Revista Brasileña de Plantas Medicinales, v. 14, n. 2, p. 327-334, 2012.

SERRÃO, D. Busca sabiduría, comparte conocimiento. Cofanor, 2010.

SMITH, B.; TANG, K.; NUTBEAM, D. WHO health promotion glossary: new terms. Health Promotion International, v. 21, n. 4, p. 340-345, 2006.

TARTUCE, T. J. A. Métodos de búsqueda. Fortaleza: UNIC - Educación Superior, 2006.

TURA, L. Paradigmas de promoción y prevención de la salud. In: LOPES, M.; MENDES, F.; MOREIRA, A. (Coord.). Salud, educación y representaciones socialies: ejercicios de diálogo y convergencia. Coimbra: Formasau, 2009. p. 151-161.

UNESCO - UNITED NATIONS EDUCATIONAL, SCIENTIFIC AND CULTURAL

ORGANIZATION. Sexta conferencia internacional de adultos: hito de acção de Belém. Brasília, DF, 2010.

VAZ, P.; POMBO, M.; FANTINATO, M.; PECLY, G. El factor de riesgo en los medios. Botucatu. Interface, v. 11, n. 21, p. 145-153, 2007.

WHO - WORLD HEALTH ORGANIZATION. The Nairobi call to action for closing the implementation gap in health promotion. Geneva, 2009. 